\title{
Electrode-separated Quartz Crystal Microbalance for Smart-sensor Mounting Application
}

\author{
Jun Sakaguchi, Masayuki Sohgawa, and Takashi Abe* \\ Graduate School of Science and Technology, Niigata University, \\ 8050 Ikarashi 2-no-cho, Nishi-ku, Niigata 950-2181, Japan
}

(Received July 27, 2017; accepted October 18, 2017)

Keywords: quartz crystal microbalance, separated electrode, crystallographic $x$-axis

Quartz crystal microbalances (QCMs) have found applications as popular chemical and biological sensing tools. However, their current device geometry prevents their commercialization for industrial use. In this study, we propose a new disposable QCM sensor with an electrode only on the sensing side, making it suitable for sensor mounting. In this sensor system, the excitation electrode is positioned on a glass plate separate from the QCM. Such electrode configurations are suitable for sensor applications in flow cell systems in preference to common QCM systems because QCM mounting on the flow cell and the electrical interconnections between the QCM and the oscillation circuit are simple. The optimized configurations of the electrode diameter, separation distance, and orientation relative to the crystallographic $x$-axis of the plate are revealed for the first time. The proposed QCM can be applied to realize a disposable QCM-based biochip or hazardous gas sensor located inside a flow tube.

\section{Introduction}

Quartz crystal microbalances (QCMs) are widely popular chemical and biological sensing tools that are often used in monitoring interactions between biochemical molecules. ${ }^{(1-6)}$ The common QCM has electrodes located on both sides of an AT-cut quartz crystal. Upon application of a voltage pulse between these electrodes, a thickness shear mode (TSM) is generated as the principle vibration mode. Thus, any mass change occurring on the surface of the QCM is proportional to the frequency change. ${ }^{(7)}$ This principle is advantageous for biosensing since it allows the measurement of binding reactions such as antigen-antibody reactions. ${ }^{(2)}$ The advantages of the QCM include real-time and label-free detection of molecular adsorption or desorption. Another advantage of the QCM system is that it is based on a simple electronic circuit, except for its packaging; the packaging is one of its biggest obstacles to industrial use.

Regarding this concern, we previously reported a new type of QCM, an antiparallel coupled resonator (ACR), which has two electrodes located on one side of an AT-cut quartz crystal

${ }^{*}$ Corresponding author: e-mail: memsabe@eng.niigata-u.ac.jp

http://dx.doi.org/10.18494/SAM.2018.1712 
with a sensing electrode positioned on the other side of the crystal. ${ }^{(8,9)}$ It has been speculated that two oscillators are formed between the electrodes for exciting the TSM and the sensing electrode, thereby leading to opposite phase coupling. Because of opposing electric fields, the resonator displacements occur in opposite directions. With our proposed method, the difficulty in connecting the wiring to the electrodes located on the sensing side of the flow cell is solved. Our results suggest that the ACR is an attractive alternative to the standard QCM.

Although the proposed QCM sensor solves the difficulty of sensor mounting within the flow cell, the ideal method suitable for smart packaging is wireless connection of the electrodes.

Historically, early models of quartz resonators utilized solid electrodes positioned close to the crystal surface. ${ }^{(10)}$ With this mounting approach, a small amount of free motion of the crystal relative to the electrodes causes occasional changes in the frequency, which is problematic. Thus, the use of metal deposited electrodes on the crystal is considerably more preferable. However, if QCM use is limited to short time intervals without free motion affecting the support of the crystal, the former method becomes attractive because of the simplicity of mounting. Indeed, crystal blanks are tested to evaluate the quality before plating in the manufacturing process.

Several approaches have been proposed to achieve wireless oscillation of the AT-cut quartz resonator for use in QCMs. ${ }^{(11-13)}$ In such approaches, the quartz plate is separated from the oscillation circuit. From the viewpoint of disposable use, a QCM with a transmitter is out of the question. ${ }^{(14)}$ On the other hand, an electrodeless QCM sensor is considered a promising approach to achieving wireless sensing of mass changes. ${ }^{(1)}$ In such a sensor, the exciting electrodes are removed from both surfaces of the quartz crystal plate and platinum electrodes are set on both sides of the crystal plate approximately $30 \mathrm{~mm}$ apart. This approach is disadvantageous from the viewpoint of device geometry; ideally, one side of the crystal must be free for sensing.

In this context, researchers have recently reported a wireless-electrodeless QCM (WE-QCM) sensor. ${ }^{(13)}$ This sensor uses an antenna for wireless actuation. The sensor's sensitivity is high because all the electrodes and wires are removed from the crystal. While the method appears ideal for QCM applications, the sensor system requires special circuits for actuating the WEQCM. In addition, it is to be noted that the patterning of gold electrodes is not a disadvantage for most biochemical applications because the biomolecules are immobilized over a selected area.

In terms of practicality, a simple oscillator circuit widely adapted in a common QCM setup $^{(15)}$ is expected to provide low-cost sensing systems accessible to all users. The overall simplification of the system is crucial to realizing a QCM-based portable medical diagnostic device.

In this study, we demonstrate an ACR-type QCM that can be used as an alternative to wireless QCM sensors. From the viewpoint of device geometry, the ACR is suitable for application to a QCM-embedded disposable sensor chip because one side of the crystal is free for sensing use. The proposed sensor is also suitable for gas sensing because it can detect hazardous gas flowing through a glass tube without the need for any electrical interconnections. 


\section{Experimental Methods}

\subsection{Sensor design}

Figure 1 shows the experimental setup used in this study. The AT-cut quartz crystal used in our experiment had a thickness of $100 \mathrm{~mm}$ and a diameter of $12 \mathrm{~mm}$. The quartz crystal was purchased from Seiko EG\&G (Chiba, Japan). The crystal was polished for mass production of frequency control devices, and the surface roughness was below $2 \mathrm{~nm}$.

Figure 1(a) shows the geometry of the electrodes. The circular electrode (sensing electrode) consisted of a 100-nm-thick gold layer, below which a 30-nm-thick chromium adhesion layer was patterned on one side of the crystal plate. These layers were deposited with an evaporator (VPC 260F, ULVAC KIKO).

Figure 2 shows an example of the fabricated sensor. In the study, the sensing-electrode diameter was varied from 2 to $12 \mathrm{~mm}$. The two excitation electrodes with diameters of $2 \mathrm{~mm}$ were configured with a $0.1 \mathrm{~mm}$ electrode gap between them that was aligned at a specific angle to the crystallographic $x$-axis (Fig. 1). The gap distance is important because the resonator is a coupled resonator composed of two resonators. ${ }^{(8)}$ The geometry is similar to that of a lateral field excited (LFE) sensor ${ }^{(16-18)}$. However, the gap distance differs by one order of magnitude (1-3.5 mm) compared with that of the ACR-type QCM $(0.1 \mathrm{~mm})$, and it has no sensing electrode on the other side; thus, the driving mechanism is not the same as that of the ACR-type QCM. The LFE sensor works as a thickness field excitation sensor when immersed in an electrolyte solution. ${ }^{(18)}$ Under this condition, the driving mechanism is similar to that of the ACR-type QCM. The details of the ACR-type QCM have already been reported in a previous article. ${ }^{(8)}$

In our study, we also evaluated the excitation-electrode-gap angle dependence of the resonance frequency. The excitation electrodes, consisting of a 100-nm-thick gold layer and 30 -nm-thick chromium adhesion layer, were fabricated on a glass plate. Further, the separation between the quartz plate and the glass plate was varied from 0 (=contact) to $0.5 \mathrm{~mm}$ by means of spacers. The resonance frequency was $\sim 16.8 \mathrm{MHz}$ for all cases. This value was in agreement with the theoretical values calculated from the thickness. ${ }^{(7)}$

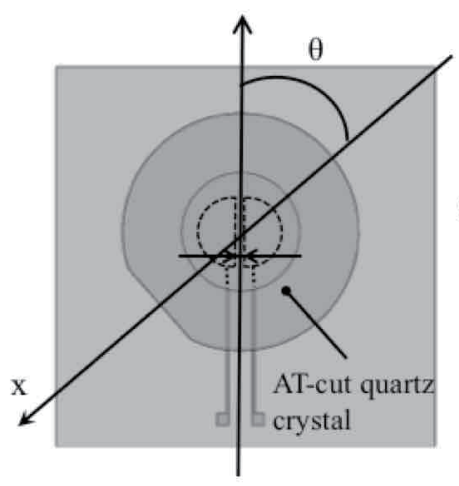

(a)

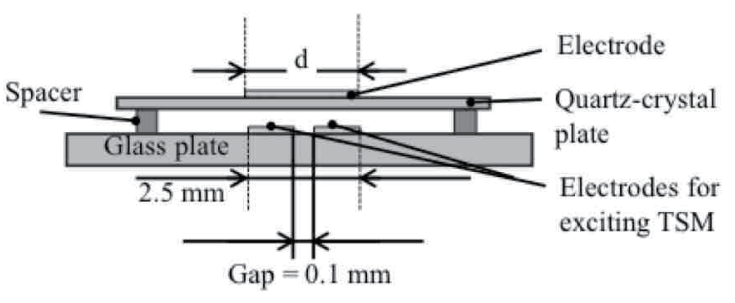

(b)

Fig. 1. (a) Top view and (b) cross-sectional view of the proposed QCM. 

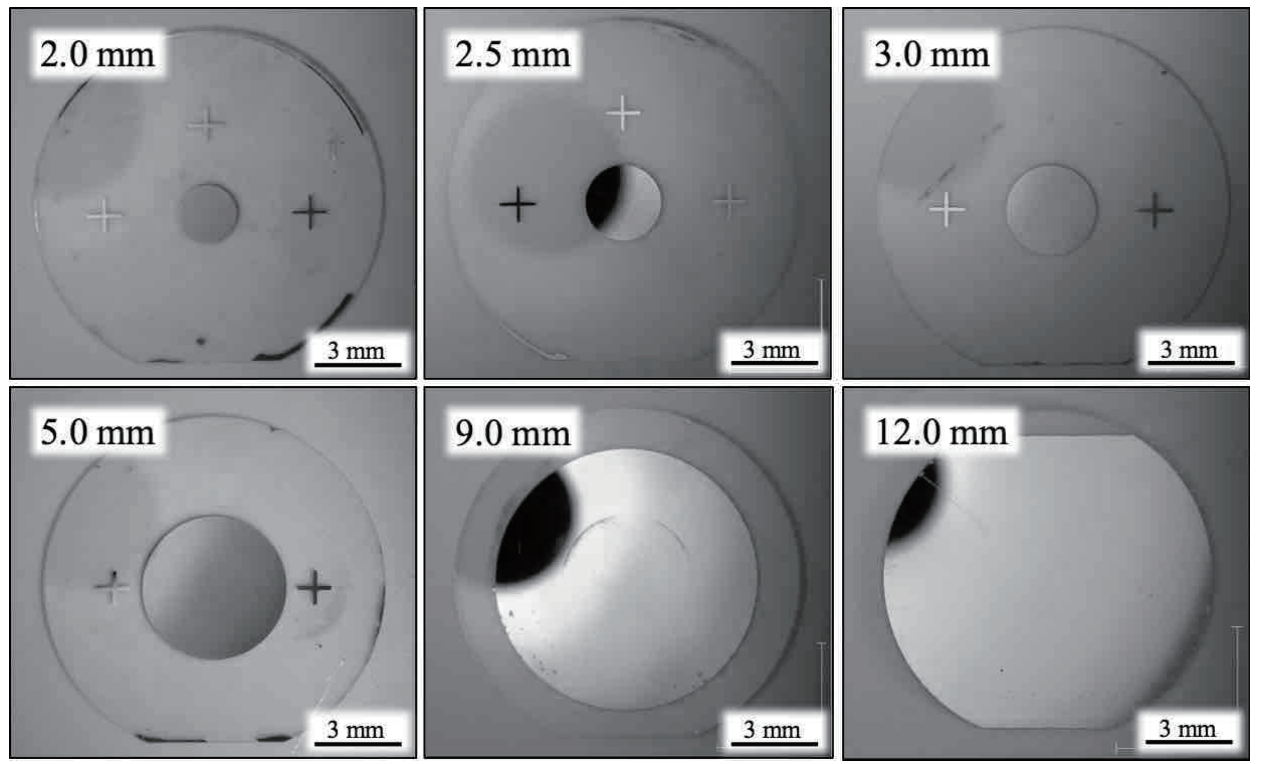

Fig. 2. Photographs of the fabricated resonators.

\subsection{Measurements}

First, the effect of the separation of the quartz plate from the glass plate on the resonance frequency was estimated using a network analyzer (MS4630B, ANRITSU) with a $\pi$-circuit jig (MA1506A) and a frequency counter (53181A, Agilent Technologies). As the oscillation circuit, a simple oscillation circuit fabricated with a CMOS inverting gate (such as 74AC04P) was adopted as a model of a widely used oscillation circuit.

Here, the gap was aligned parallel to the crystallographic $x$-axis. For the evaluation of gravimetric measurements, a photoresist (OFPR Series, TOKYO OHKA KOGYO) on the sensing electrode was patterned to cover the electrode with the photoresist. The thickness was controlled by adjusting the rotation speed of spin-coating and the viscosity of the photoresist. The density value used for the mass calculation was $1.1 \mathrm{~g} / \mathrm{cm}^{3}$. Here, it should be noted that the frequency responses to the added mass obey the Sauerbrey equation ${ }^{(7)}$ when a standard-type QCM is evaluated by this method.

Next, we evaluated the dependence of the resonant characteristics, including the frequency stability, on the patterned-electrode diameter on the quartz plate by the same method. The frequency stability was evaluated by calculating the limit of detection (LOD) for $1 \mathrm{~m}$. We multiplied the standard deviation of the resonance frequency by three when we calculated the LOD. The resonance frequency was normalized by the resonance frequency at $\theta=0$. Finally, the electrode-gap-angle dependence on the normalized frequency was evaluated using a frequency counter. In this phase of the study, the diameter of the patterned electrode used was 2.5 $\mathrm{mm}$. All the measurements were carried out at room temperature. 


\section{Results and Discussion}

\subsection{Frequency stability vs separation}

Figure 3 shows the frequency stability as a function of the separation between the quartz and glass plates. The electrode diameter is $2.5 \mathrm{~mm}$. The measured frequencies are stable within $0.4 \mathrm{~Hz}$ for separations up to $200 \mu \mathrm{m}$. From the gravimetric measurements (Fig. 4), the limit of detectable mass is estimated to be about $0.1 \mathrm{ng}$. The sensitivity per unit area is $0.4 \mathrm{ng} / \mathrm{Hz} \mathrm{cm}{ }^{2}$ for all separation distances. Since the mass detection limit of the common QCM ranges from 0.1 to $1 \mathrm{ng}$, we can conclude that the proposed sensor has sufficient sensitivity. The frequency

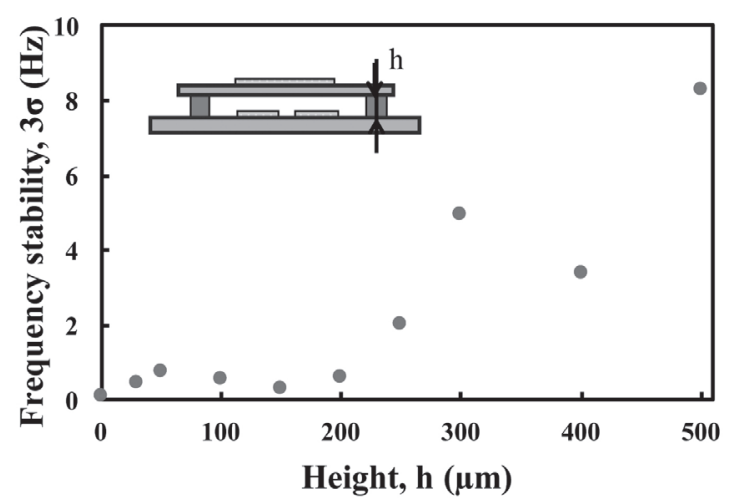

Fig. 3. Frequency stability of the QCM at various separation distances between the quartz and glass plates.
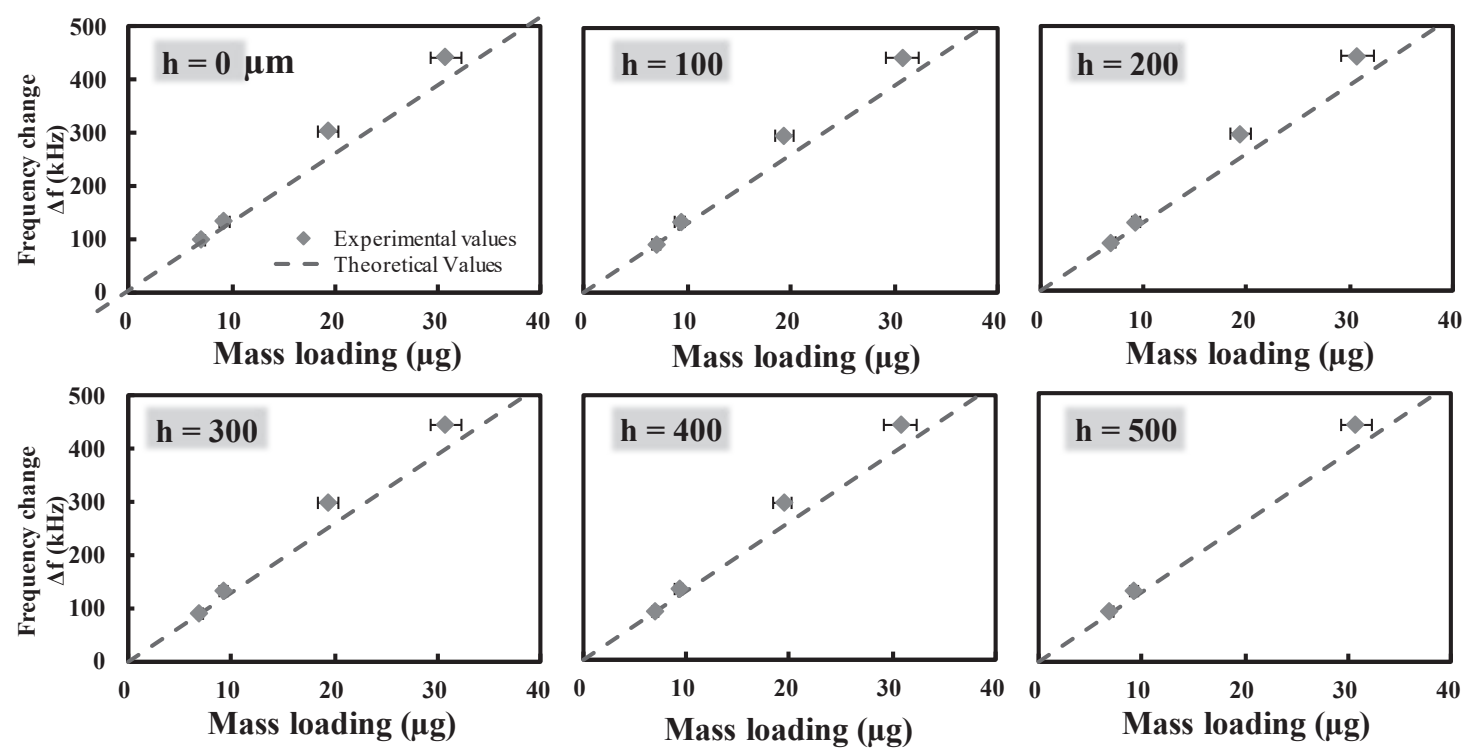

Fig. 4. Frequency response vs added mass for various separation distances between the quartz and glass plates. 
response versus mass loading shows a linear correlation, except for large mass loadings. This deviation from the theoretical prediction is explained by the difference between Young's modulus and the density of the quartz crystal and that of the photoresist. As is well known, the Sauerbrey equation is not accurate in the high-mass-density regime. ${ }^{(19)}$

\subsection{Resonance-frequency dependence on crystallographic orientation}

Figure 5 shows the dependence of the resonance frequency on the crystallographic orientation. In this study, the frequency was evaluated by varying the electrode-gap angle with respect to the crystallographic $x$-axis of the plate. This angle $\theta$ was changed from 0 to $180^{\circ}$ in the counterclockwise direction as shown in Fig. 1(a). The separation distance is 0 in this evaluation. The setting $\theta=0$ indicates that the electrode gap is parallel to the crystallographic $x$-axis of the plate. It is important to note that the resonance frequency is relatively stable along this direction. Interestingly, a sudden frequency change toward higher frequencies is observed around at $\theta=45-60^{\circ}$ and $\theta=120-135^{\circ}$. The resonance frequency depends on the crystal thickness and acoustic wave velocity, which in turn depends on the crystallographic orientation. Therefore, the sudden frequency change is explained by a change in the direction of the vibration. This result is reasonable when we consider that there are three vibration modes that depend on the electric field direction. ${ }^{(16,18)}$ The mode change discovered in this work is expected to apply to this dual-mode QCM sensor on a single chip.

From the discussion, we can conclude that, in accordance with the resonance-frequency dependence on crystallographic orientation, the electrode gap should be parallel to the crystallographic $x$-axis of the plate to ensure sensor stability.

\subsection{Frequency dependence on electrode diameter}

Figure 6 shows the dependence of the frequency stability on the sensing-electrode diameter. The stability is within $0.4 \mathrm{~Hz}$ in the diameter range from 1 to $9 \mathrm{~mm}$. It appears that the

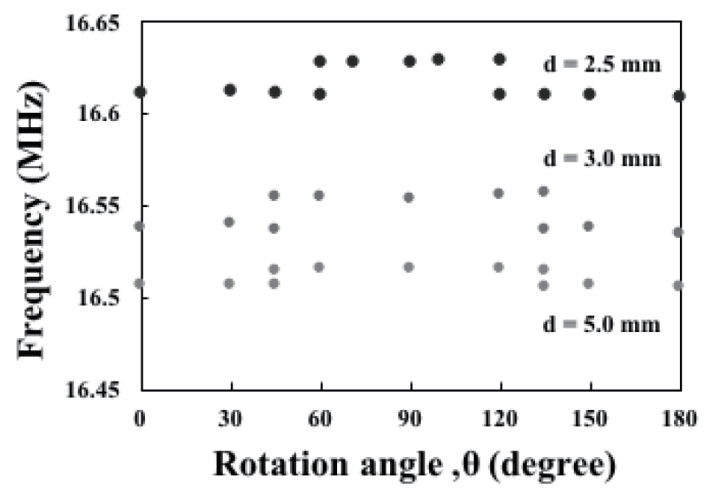

(a)

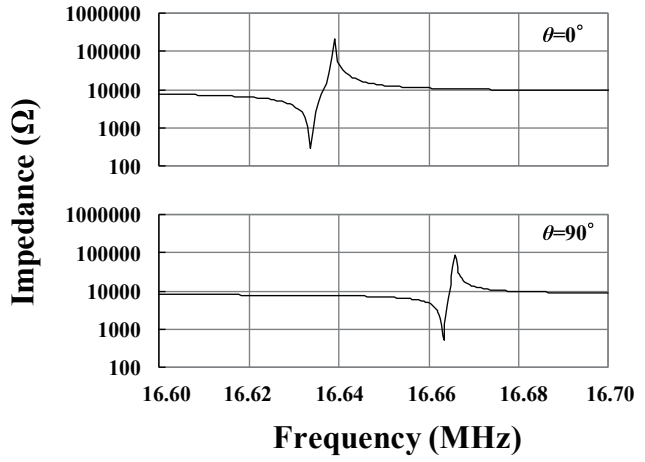

(b)

Fig. 5. Dependence of resonance frequency of the QCM on (a) the electrode-gap orientation angle and (b) examples of the impedance spectra. 
frequency is stable for a 12-mm-diameter QCM; however, there are two main vibrational modes in the impedance spectra (Fig. 7). Large electrode diameters are not suitable for coupling of vibrations because the electric field is not confined within the selected volume. The vibration mode caused by the coupling of the two resonators is more stable when the electric field is confined around the gap; the confinement of electric field strengthens the vibration mode coupling. This strengthening effect can be observed in the dependence of the resonancefrequency change on the crystal orientation. The sudden frequency change is not observed at $\theta$ $=45$ and $135^{\circ}$ for small electrode diameters, as can be observed in Fig. 5 .

In summary, our results indicate that our proposed wireless QCM can be utilized for various sensing applications.

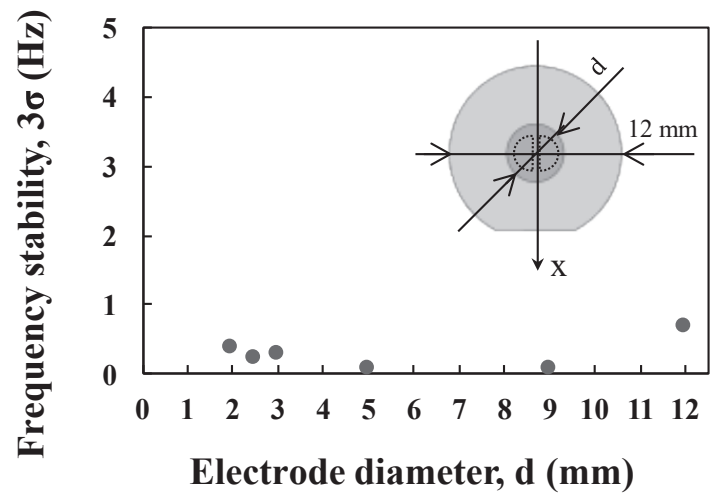

Fig. 6. Frequency stability of the QCM for various diameters of the sensing electrode.
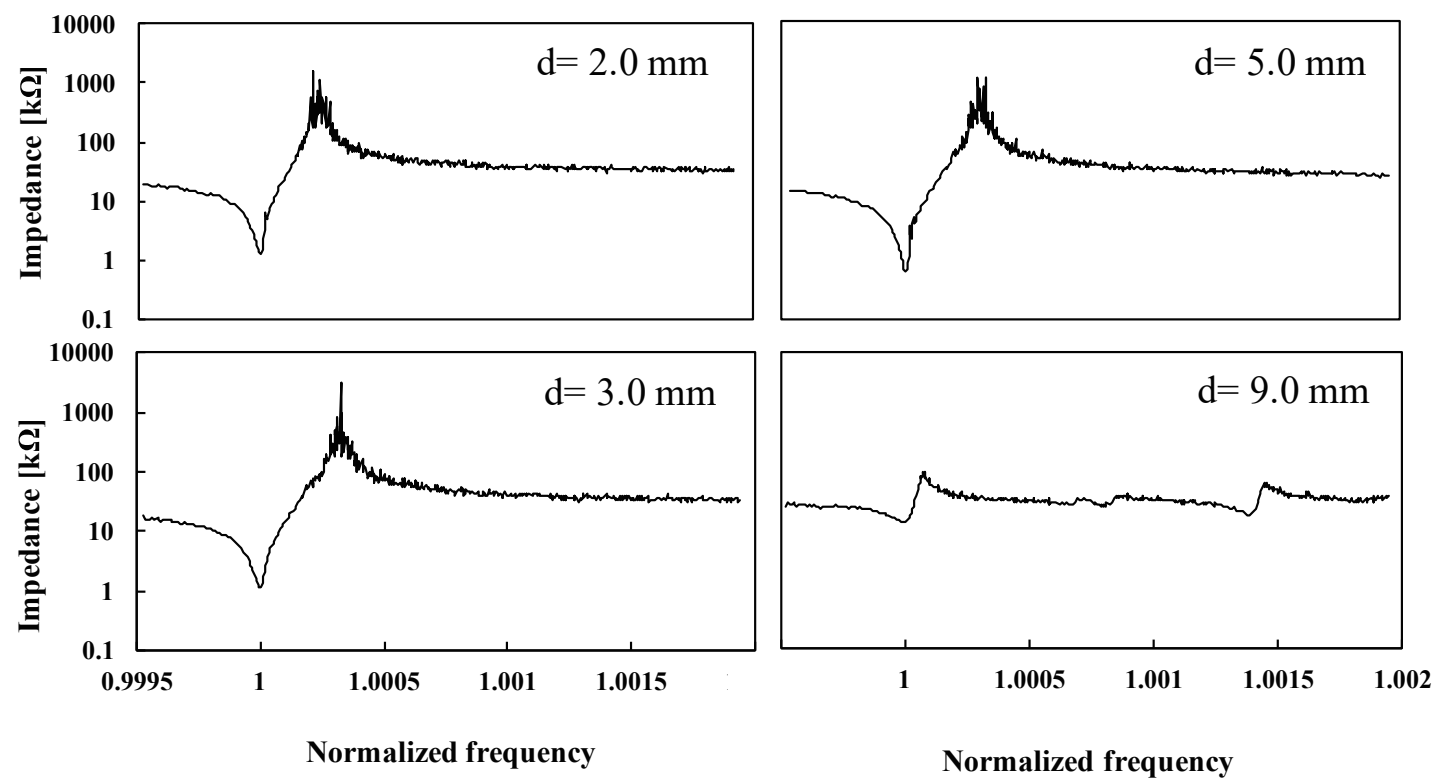

Fig. 7. Impedance spectra of the QCM for different diameters of the sensing electrode. 


\section{Conclusions}

In this study, we proposed a new disposable QCM sensor with an electrode on the sensing side and no electrode on the other side, making it suitable for sensor mounting (for example, in flow tubes). In this sensor system, the excitation electrode is located on a glass plate that is separate from the QCM. For a separation distance of $500 \mu \mathrm{m}$, the sensor exhibits oscillations with a standard oscillation circuit constructed with a CMOS inverting gate, as well as a linear response to mass loading. The frequency stability lies within $0.4 \mathrm{~Hz}$ below a separation of 200 $\mu \mathrm{m}$. We also determined that the electrode gap should be parallel to the crystallographic $x$-axis of the plate to ensure sensor stability. The proposed QCM sensor can be utilized as a disposable sensor that is suitable for industrial use because of its ease of use and construction.

\section{Acknowledgments}

This work was supported in part by a Grant-in-Aid for Scientific Research from the Ministry of Education, Science, Sports and Culture of Japan (16K13649).

\section{References}

1 W. H. King: Anal. Chem. 36 (1964) 1735.

2 H. Muramatsu, M. D. Dicks, E. Tamiya, and I. Karube: Anal. Chem. 59 (1987) 2760.

3 Y. Okahata, Y. Matsunobu, K. Ijiro, M. Mukae, A. Murakami, and K. Makino: J. Am. Chem. Soc. 114 (1992) 8299.

4 W. P. Carey, K. R. Beebe, B. R. Kowalski, D. L. Illman, and T. Hirschfeld: Anal. Chem. 58 (1986) 149.

5 K. Ema, M. Yokoyama, T. Nakamoto, and T. Moriizumi: Sens. Actuators 18 (1989) 291.

6 J. X. Liang, J. Zhang, P. Wang, C. C. Liu, S. Qiu, and T. Ueda: Sens. Mater. 28 (2016) 201.

7 G. Sauerbrey: Z. Phys. 155 (1959) 206.

8 T. Abe and H. Kato: Anal. Chem. 79 (2007) 6804.

9 T. Abe and H. Kato: J. Micromech. Microeng. 19 (2009) 094019.

10 W. A. Marrsion: Bell Syst. Tech. J. XXVII (1948) 510.

11 T. Nomura, F. Tanaka, T. Yamada, and H. Itoh: Anal. Chim. Acta 243 (1991) 273.

12 J. Rabe, V. Seidemann, and S. Buettgenbach: Sens. Mater. 15 (2003) 381.

13 H. Ogi, K. Motoshisa, T. Matsumoto, K. Hatanaka, and M. Hirano: Anal. Chem. 78 (2006) 6903.

14 X. Zhou, J. Zhang, T. Jian, X. Wang, and Z. Zhu: Sens. Actuators, A 135 (2007) 209.

15 J. R. Vig: Tech. Rep. SLCET-TR88-1 (1991).

16 Y. Hu, L. A. French, K. Radecsky, M. P. da Cunha, P. Millard, and J. F. Vetelino: IEEE Trans. Ultrason. Ferroelectr. Freq. Control. 51 (2004) 1373.

17 Y. Hu, W. Pinkham, L. A. French, D. Frankel, and J. F. Vetelino: Sens. Actuators, B 108 (2005) 910.

18 W. Wang, C. Zhang, Z. Zhang, Y. Liu, and G. Feng: Appl. Phys. Lett. 93 (2008) 242906.

19 J. Janata: Principles of Chemical Sensors (Springer, New York, 2009). 